\title{
Método "fingerprinting" para identificação de fontes de sedimentos em bacia hidrográfica rural
}

\author{
Jean P. G. Minella ${ }^{1}$, G ustavo H. Merten ${ }^{1} \&$ Robin T. Clarke ${ }^{1}$
}

\begin{abstract}
RESU MO
Este trabalho se refere a um estudo de identificação de fontes de sedimentos, realizado em uma bacia rural localizada em uma região montanhosa, no sul do Brasil. A bacia representa um ecossistema típico de exploração agrícola, realizado por pequenos agricultores que cultivam fumo em áreas marginais, com conseqüências severas aos recursos naturais. A identificação das fontes de sedimentos, baseada na análise dos sedimentos transportados em suspensão, possibilita avaliar as áreas que contribuem para a sua produção e fazer inferências sobre o potencial poluidor desses sedimentos. A metodologia utilizada foi a técnica "fingerprinting", através da qual foram exploradas as principais limitações da metodologia e as potencialidades na identificação das fontes de sedimentos. A classificação possibilitou a identificação da contribuição relativa das fontes, considerando-se preponderantes as lavouras e as estradas. $0 \mathrm{~s}$ resultados mostraram que a contribuição das duas fontes foi variável no tempo e a proporção relativa da contribuição de cada fonte variou de acordo com o manejo e a cobertura do solo que ocorreu nas lavouras e das obras de manutenção realizadas nas estradas. A contribuição das fontes mostrou que as lavouras e as estradas contribuíram com 64 e 36\%, respectivamente.
\end{abstract}

Palavras-chave: produção desedimentos, aproximação "fingerprinting", monitoramento emodelagem de baciashidrográficas

\section{Fingerprinting method for identification of sediment sources in a rural watershed}

\begin{abstract}
In this study, the fingerprinting method was used to identify sediment sources in a small rural watershed located in a mountainous region in southern Brazil. This watershed represents a typical agricultural ecosystem, in which smallhold farmers cultivate tobacco in marginal areas, with severe consequences to the natural resources. The identification of sediment sources, based on analysis of sediments transported in suspension, enables an evaluation of which areas are truly contributing to sediment production and, therefore, to make inferences about the polluting potential of these sediments. The study considered the main limitations of this method, as well as its potential in the identification of sediment sources. Through classification, it was possible to identify the relative contribution of these different sources, with fields and roads being predominant. Results showed that the contribution from these two sources varied over time and that the relative proportion of the contribution from each source varied according to management and soil cover in the fields and maintenance work performed in the roads. The average contribution from fields and roads was 64 and $36 \%$, respectively.
\end{abstract}

Key words: sediment yield, composite fingerprinting technique, small watersheds monitoring and modeling

1 Instituto de Pesquisas Hidráulicas/U FRGS, Av. Bento Gonçalves 9500, CEP 90650-001, Porto Alegre, RS. Fone: (51) 3308-6325. E-mail: jminella@gmail.com; merten@iph.ufrgs.br; clarke@iph.ufrgs 


\section{INTRODUÇÃO}

No sul do Brasil o fumo (Nicotina tabacum) é cultivado por pequenos agricultores em solos considerados de baixa aptidão agrícola, devido à alta declividade e à sua pouca profundidade. A este aspecto se soma o manejo inadequado praticado pelos agricultores em virtude do intenso revolvimento do solo realizado para o cultivo do tabaco, durante o período de maior erosividade das chuvas. A combinação desses fatores favorece o processo da erosão hídrica que tem, como consequiências, o empobrecimento dos solos e a elevada produção de sedimentos. Para atenuar o problema e aliviar a visível pobreza rural, estão sendo implantados programas financiados por recursos públicos e internacionais que têm, como estratégia principal, a introdução de práticas conservacionistas que objetivem principalmente a redução da erosão hídrica. Os impactos da implantação das práticas conservacionistas sobre os recursos naturais têm sido avaliados através de programas de monitoramento de bacias hidrográficas rurais, estudando-se aspectos referentes à hidrologia, à qualidade da água e à produção de sedimentos (Merten \& Minella, 2003).

Atualmente, grande atenção se volta aos estudos direcionados para o desenvolvimento de métodos que possibilitem a identificação dessas fontes de produção (Horowitz, 1991; Symader \& Strunk, 1992; Collins \& Walling, 2002). Os sedimentos que transitam em suspensão na calha fluvial têm origem em diferentes fontes. A proporção com que cada fonte contribui na mistura é variável no tempo e no espaço, conseqüência dos processos erosivos que estão em curso na bacia de contribuição. Um dos métodos mais promissores que se têm destacado pela eficiência, é a técnica "fingerprinting" (Peart, 1995; Collins et al., 1997; Walling et al.,1999; Walling \& Collins, 2000; Collins \& Walling, 2002), que se baseia no princípio de que os sedimentos em suspensão mantêm algumas de suas propriedades geoquímicas adquiridas nas fontes, sendo essas propriedades, então, utilizadas como traçadores.

Considerando que os sedimentos são provenientes de diferentes fontes (estradas, lavouras, pastagens canal fluvial etc.), é provável que, no processo de monitoramento, seja possível não somente quantificar a variação temporal da produção de sedimentos, mas, também, identificar a origem dessas fontes, com que se espera obter resultados mais conclusivos em relação ao efeito das práticas conservacionistas na redução da produção de sedimentos. Com este fim, este trabalho foi realizado com de avaliar a técnica "fingerprinting" para utilização na identificação da proveniência dos sedimentos erodidos em uma bacia hidrográfica rural, com grande intensidade do processo erosivo e produção de sedimentos.

\section{MATERIAL E MÉTODOS}

\section{Caracterização da bacia}

Este trabalho foi realizado no sul do Brasil (Figura 1) em uma pequena bacia hidrográfica rural situada nas cabeceiras do Arroio Lajeado Ferreira, tributário do Rio
Taquari. A área da bacia monitorada possui 119 ha, porém os estudos de identificação de fontes de produção de sedimentos foram realizados em uma sub-bacia de 57 ha, denominada SB1, conforme pode ser identificado na Figura 1; nela, o relevo predominante é ondulado (declividade média 7\%) e os solos são pouco desenvolvidos (profundidade média de $0,50 \mathrm{~m}$ ) predominando as unidades taxonômicas do tipo Argilossolos, Neossolos e Cambissolos. A drenagem da SB1 apresenta declividade média de $8 \%$ e um tempo de concentração de $11 \mathrm{~min}$.

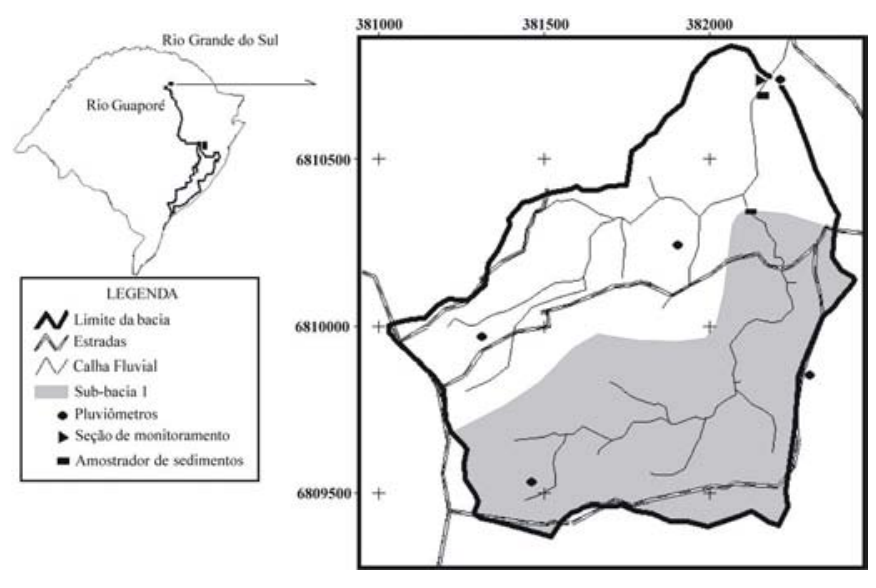

Figura 1. Localização da bacia estudada

Os diferentes usos do solo na SB1 são lavouras anuais cultivadas com tabaco $31 \%$, pastagem $29 \%$, pousio $37 \%$ e estradas e caminhos não pavimentados, $3 \%$. O sistema de manejo da cultura do tabaco é realizado de forma tradicional cujo solo é preparado com uso de arado com tração animal nos períodos de maior erosividade das chuvas que correspondem aos meses de agosto, setembro e outubro; esta condição, aliada à ausência da mata ciliar, favorece o processo de erosão hídrica nas lavouras e a produção de sedimentos quantificando-se, para o ano de 2002, uma produção de sedimentos aproximada de $200 \mathrm{t} \mathrm{km}^{-2}$ ano $^{-1}$ (Merten \& Minella, 2003).

\section{Coleta das amostras das fontes de sedimentos}

A escolha das fontes de produção de sedimentos foi definida após um levantamento realizado na SB1 para identificar os processos erosivos que estavam ocorrendo na área; desta forma, as fontes de sedimentos selecionadas foram lavouras antigas e recentes de tabaco, pastagem, pousio, estradas não pavimentadas e canal fluvial. As amostras de solo dessas fontes foram coletadas na camada superior do solo $(0-0,05 \mathrm{~m})$. Seis amostras foram coletadas para representar cada fonte e cada amostra se compunha de 10 sub-amostras. A quantidade de material coletado para cada amostra foi de aproximadamente $0,5 \mathrm{~kg}$; foi secada à sombra e posteriormente peneirada em peneira de $2 \mathrm{~mm}$ este material encaminhado para realização das análises físicas e químicas.

\section{Coleta das amostras dos sedimentos em suspensão}

Avaliações das descargas líquidas, sólida e qualidade 
de água vêm sendo realizadas em uma seção do arroio Lajeado Ferreira no qual se instalou uma calha do tipo Parshall; já as coletas de sedimentos em suspensão, utilizadas para a identificação das fontes de sedimentos, foram obtidas na seção do arroio da sub-bacia SB1, conforme a Figura 1. Utilizou-se, na coleta dessas amostras, um equipamento que armazena a amostra em galões plásticos com capacidade para $40 \mathrm{~L}$; o amostrador construído se assemelha ao amostrador de nível ascendente (FISP, 1961), porém com algumas modificações importantes: a) em substituição às garrafas, foi acoplado um galão de $40 \mathrm{~L}$ para onde o fluxo (amostra) é direcionado por meio de mangueiras; b) as amostras coletadas nas diferentes profundidades são misturadas no galão, uma vez que o importante é a coleta de uma amostra representativa do perfil vertical; c) o amostrador coleta durante a subida e a descida da onda de cheia; e d) os espaçamentos entre as tomadas de água são de $7 \mathrm{~cm}$.

O período de monitoramento dos sedimentos em suspensão foi de abril até outubro de 2002, que abrangeu as principais mudanças de uso do solo na bacia, tais como: colheita do fumo da safra 2002, pousio invernal das áreas cultivadas na safra 2002, preparo do solo para o fumo da safra 2003, retificação de estradas e desmatamento de áreas transformadas em lavouras para a safra do fumo de 2003, neste período também foram amostrados os eventos de maior magnitude, no total de 10.

\section{Caracterização física e química das amostras}

As análises granulométricas dos sedimentos do material obtido nas fontes e dos sedimentos em suspensão, foram determinadas utilizando-se o método da peneiragem e pipetagem (Carvalho, 1994). As concentrações dos elementos químicos dos sedimentos nas fontes e em suspensão foram determinadas segundo os métodos: a) Teores totais de N, P, $\mathrm{K}, \mathrm{Ca}, \mathrm{Na}, \mathrm{Mg}, \mathrm{Cu}, \mathrm{Pb}, \mathrm{Cr}, \mathrm{Co}, \mathrm{Zn}, \mathrm{Ni}, \mathrm{Fe}, \mathrm{Mn}$ e $\mathrm{Al}$, com extração por digestão de ácido sulfúrico (Tedesco et al., 1995); b) C orgânico total, pelo método de Walkley-Black (Tedesco et al., 1995); c) $\mathrm{Al}^{+3}$ extraído por $\mathrm{KCl}(1 \mathrm{M})$ e titulação com $\mathrm{NaOH}$ (Tedesco et al., 1995); d) Óxidos de Fe e $\mathrm{Mn}$, por extração com Ditionito-Citrato Bicarbonato de sódio (Inda Júnior, 2002); e) Óxido de Fe com extração com Oxalato de Amônio ácido (Inda Júnior, 2002).

Para realizar as correções das concentrações dos elementos químicos determinados, os quais são dependentes da granulometria dos sedimentos em suspensão, utilizou-se a Eq. 1 (Horowitz, 1991).

$$
Z_{\mathrm{si}}=\mathrm{C}_{\mathrm{o}}\left[100 /\left(100-\mathrm{F}_{\mathrm{ars}}\right)\right]
$$

em que $Z_{\mathrm{si}}$ é a concentração corrigida das fontes e do elemento químico analisado (i), $\mathrm{C}_{\mathrm{o}}$ é a concentração média original do elemento químico analisado e $\mathrm{F}_{\text {ars }}$ é a porcentagem da fração maior que $0,063 \mathrm{~mm}$ das amostras da fonte.

\section{Discriminação das fontes de sedimentos}

Duas características dos sedimentos erodidos são importantes para que uma propriedade geoquímica seja efetiva como elemento traço; primeiro, que apresente diferenças significativas na sua concentração entre as fontes a serem identificadas; a segunda característica é que haja manutenção desta propriedade durante a remoção e o transporte dos materiais das fontes, até a calha do rio.

A primeira etapa para a identificação das fontes de sedimento foi a análise dos materiais de todas as fontes, com o objetivo de estabelecer se havia diferença significativa entre as fontes e quais parâmetros possibilitariam separá-las; para este procedimento foi utilizado um teste não-paramétrico, denominado teste-H ou Kruskall-Wallis (Walling \& Collins, 2000). O teste permite testar uma hipótese nula em que $\mathrm{k}$ amostras aleatórias independentes são provenientes de uma mesma população (Eq. 2).

$$
\mathrm{H}=\frac{12}{\mathrm{n}(\mathrm{n}+1)} \sum_{\mathrm{s}=1}^{\mathrm{k}} \frac{\mathrm{R}_{\mathrm{s}}^{2}}{\mathrm{n}_{\mathrm{l}}}-3(\mathrm{n}+1)
$$

em que $R_{s}$ se refere à soma dos ranks ocupados pela fonte $s$, sendo $\mathrm{n}_{1}$ o número de observações da fonte $\mathrm{s}, \mathrm{n}$ a soma dos $\mathrm{n}_{1}$ 's e $\mathrm{k}$ o número de fontes.

Aplicou-se, para cada variável previamente analisada, o teste verificando-se sua capacidade em discriminar as fontes ao se testar as diferenças para uma variável i qualquer para 3 fontes; por exemplo, tem-se 6 amostras $\left(n_{1}=6\right)$ coletadas para cada fonte; a hipótese nula $\left(\mathrm{H}_{0}\right)$ a ser testada é que as populações são idênticas e a hipótese alternativa $\left(\mathrm{H}_{1}\right)$ é de que as populações diferem.

Quando o valor de $\mathrm{H}$ calculado exceder o valor crítico de $\mathrm{H}$, com k-1 graus de liberdade e grau de significância 5\%, dir-se-á que a hipótese nula deve ser rejeitada, ou seja, a probabilidade é pequena (5\% ou menos) de se observar diferenças das amostras, se as observações são provenientes da mesma população, ou seja, a variável apresenta capacidade discriminante entre as fontes.

A escolha das variáveis que apresentam capacidade de discriminação entre as fontes de sedimentos não é o suficiente para a aplicabilidade do método. Existe um conjunto mínimo de variáveis que maximiza a discriminação e diminui a dimensionalidade; a verificação foi realizada por meio de uma análise de variância multivariada; assim, a segunda etapa foi aplicar uma análise de variância multivariada para as propriedades selecionadas na primeira etapa, verificando-se qual o melhor conjunto de variáveis que possibilita separar as fontes estatisticamente e qual é o mínimo de variáveis necessárias. $\mathrm{O}$ conjunto de propriedades a serem escolhidas deveria refletir diferentes controles e comportamentos do processo de emissão de sedimentos para maximizar o grau de discriminação fornecido pelo conjunto de variáveis.

O objetivo foi encontrar uma composição de variáveis através das quais se apresentasse a possibilidade de se realizar uma discriminação ótima entre os grupos individuais de fontes (Johnson \& Wichern, 1992); isto foi realizado com o auxílio do programa Genstat ${ }^{@}$, com um algoritmo do tipo "stepwise", baseado na minimização do parâmetro Wilks' lambda $\left(\Lambda^{*}\right)$ componente de uma análise de variância multivariada. 
Para as amostras coletadas na bacia foram organizadas as $\mathrm{g}$ diferentes populações na forma vetorial

Fonte 1: $\mathrm{X}_{11}, \mathrm{X}_{12}, \ldots, \mathrm{X}_{1 \mathrm{n} 1}$

Fonte 2: $X_{21}, X_{22}, \ldots, X_{2 n 2}$

Fonte $\mathrm{g}: \mathrm{X}_{\mathrm{g} 1}, \mathrm{X}_{\mathrm{g} 2}, \ldots, \mathrm{X}_{\mathrm{gng}}$

O modelo teórico para comparação de $\mathrm{g}$ vetores médios de uma população (Johnson \& Wichern, 1992) é (Eq. 3):

$$
X_{\mathrm{lj}}=\mu+\tau_{1}+e_{\mathrm{lj}}, \quad 1=1,2, \ldots, g \text { e } j=1,2, \ldots, n_{j}
$$

donde $\mu$ é o vetor parâmetro da média total, $\tau_{1}$ representa o 1-inésimo efeito do tratamento e $\mathrm{e}_{\mathrm{lj}}$ são variáveis independentes; com isto, pode-se montar uma tabela de análise de variância multivariada, Tabela 1.

Tabela 1. Análise de variância multivariada

\begin{tabular}{lcc}
\hline Fonte de variação & $\begin{array}{c}\text { Matriz das somas dos quadrados } \\
\text { e interação dos produtos }\end{array}$ & $\begin{array}{c}\text { Grau de } \\
\text { liberdade }\end{array}$ \\
\hline Tratamento & $\mathrm{B}=\sum_{\mathrm{l}=1}^{\mathrm{g}} \mathrm{n}_{1}\left(\overline{\mathrm{x}}_{1}-\overline{\mathrm{x}}\right)\left(\overline{\mathrm{x}}_{\mathrm{l}}-\overline{\mathrm{x}}\right)^{\mathrm{T}}$ & $\mathrm{g}-1$ \\
Resíduo (erro) & $\mathrm{W}=\sum_{\mathrm{l}=1}^{\mathrm{g}} \sum_{\mathrm{j}=1}^{\mathrm{n}_{1}} \mathrm{n}_{\mathrm{l}}\left(\overline{\mathrm{x}}_{\mathrm{lj}}-\overline{\mathrm{x}}_{\mathrm{l}}\right)\left(\overline{\mathrm{x}}_{\mathrm{lj}}-\overline{\mathrm{x}}_{\mathrm{l}}\right)^{\mathrm{T}}$ & $\sum_{\mathrm{l}=1}^{\mathrm{g}} \mathrm{n}_{1}-\mathrm{g}$ \\
Total (corrigido pela média) & $\mathrm{B}+\mathrm{W}=\sum_{\mathrm{l}=1}^{\mathrm{g}} \sum_{\mathrm{j}=1}^{\mathrm{n}_{1}} \mathrm{n}_{1}\left(\overline{\mathrm{x}}_{\mathrm{lj}}-\overline{\mathrm{x}}\right)\left(\overline{\mathrm{x}}_{\mathrm{lj}}-\overline{\mathrm{x}}\right)^{\mathrm{T}}$ & $\sum_{\mathrm{l}=1}^{\mathrm{g}} \mathrm{n}_{1}-1$ \\
\hline
\end{tabular}

Os testes utilizados para se avaliar a significância de cada composição, foram o parâmetro de Wilk's Lambda $\left(\Lambda^{*}\right)$ (Eq. 4), teste F de Snedecor e a distribuição Qui-quadrada $\left(\chi^{2}\right)$ (Johnson \& Wichern, 1992).

$$
\Lambda *=\frac{|\mathrm{W}|}{|\mathrm{B}+\mathrm{W}|}
$$

em que $|\mathrm{W}|$ é o determinante da matriz da soma dos quadrados devido ao erro, e $|\mathrm{B}+\mathrm{W}|$ é o determinante da matriz da soma dos quadrados totais corrigidos pela média, sendo que B é a matriz da soma dos quadrados em razão do tratamento.

Quanto menor for $\Lambda^{*}$, a hipótese de rejeitar $\mathrm{H}_{0}$ é mais evidente, indicando que o conjunto de variáveis testadas apresenta capacidade discriminante. A comparação dos valores de $\Lambda^{*}$ determinou o melhor conjunto de variáveis que maximiza a análise discriminante e minimiza a quantidade de variáveis a serem utilizadas, para o qual os resíduos (erros) são minimizados.

Outro parâmetro para avaliar o grau de significância da capacidade discriminante das variáveis selecionadas foi uma aproximação do teste $\mathrm{F}$ para a análise multivariada; desta forma, pode-se avaliar o grau de significância a partir da tabela de distribuição do teste F, de Snedecor. A relação entre o teste $\mathrm{F}$ e a distribuição $\Lambda^{*}$ pode ser aproximada por (Johnson \& Wichern, 1992) (Tabela 2).

\section{Classificação das amostras de sedimentos em suspensão}

Considerando que os sedimentos em suspensão são compostos de uma "mistura" de solos provenientes de diferentes fontes e que existe um número considerável de elementos químicos que caracterizam as amostras, é possível realizar
Tabela 2. Aproximação do teste F para a distribuição de Wilk's

\begin{tabular}{ccc}
$\begin{array}{c}\text { No de } \\
\text { variáveis }\end{array}$ & $\begin{array}{c}\text { № de } \\
\text { grupos }\end{array}$ & Distribuição para dados normais multivariados \\
\hline $\mathrm{m} \geq 1$ & $\mathrm{n}=2$ & $\left(\frac{\sum \mathrm{n}_{1}-\mathrm{m}-1}{\mathrm{~m}}\right)\left(\frac{1-\Lambda^{*}}{\Lambda^{*}}\right) \sim \mathrm{F}_{\mathrm{m}, \Sigma \mathrm{n} \mathrm{n}-\mathrm{m}-1}$ \\
$\mathrm{~m} \geq 1$ & $\mathrm{n}=3$ & $\left(\frac{\sum \mathrm{n}_{1}-\mathrm{m}-2}{\mathrm{~m}}\right)\left(\frac{1-\sqrt{\Lambda^{*}}}{\sqrt{\Lambda^{*}}}\right) \sim \mathrm{F}_{2 \mathrm{~m}, 2(\Sigma \mathrm{n} 1 \mathrm{~m}-1)}$ \\
\hline
\end{tabular}

n é o número de variáveis, $m$ é o número de fontes e $n_{1}$ é o número de observações

uma análise multivariada que correlaciona as concentrações químicas nas fontes e nos sedimentos em suspensão (Yu \& Oldfield, 1989; Collins et al., 1997). A Eq. 5 descreve a relação numérica entre a composição química dos sedimentos em suspensão e a composição química das fontes e suas proporções.

$$
\mathrm{y}_{\mathrm{i}}=\sum_{\mathrm{s}=1}^{\mathrm{n}} \mathrm{a}_{\mathrm{is}} \mathrm{P}_{\mathrm{s}} \quad(\mathrm{s}=1,2,3, \ldots, \mathrm{n}) \mathrm{e}(\mathrm{i}=1,2,3, \ldots, \mathrm{m})
$$

donde: $y_{i}$ é a concentração do elemento químico i obtido no sedimento em suspensão, $a_{\text {is }}$ é a concentração do elemento químico i na fonte $\mathrm{s}$, e $\mathrm{P}_{\mathrm{s}}$ é a proporção com que a fonte $\mathrm{s}$ contribui para os sedimentos em suspensão.

A Eq. 5 pode ser vista como um sistema linear sobredeterminado (o número de equações é maior que o número de incógnitas), o número de equações é definido pelo número de elementos químicos selecionados na fase de discriminação das fontes e as incógnitas são as contribuições de cada fonte para os sedimentos em suspensão que se deseja obter.

A contribuição de cada fonte $\left(\mathrm{P}_{\mathrm{s}}\right)$, solução da Eq. 5, foi determinada através da função objetivo (Eq. 6) e um processo iterativo de otimização pelo método dos mínimos quadrados (Walling \& Woodward, 1995; Collins et al., 1997). O algoritmo foi compilado em planilhas Excel ${ }^{\circledR}$ e executado utilizando-se o programa "Solver" cujos resultados foram expressos em porcentagem da contribuição relativa de cada fonte para o evento analisado; além disso, foram fornecidos os erros relativos associados a cada variável para a eficiência do modelo.

$$
\sum_{i=1}^{m}\left\{\left(C_{i}-\left(\sum_{s=1}^{n} P_{s} C_{s i} Z_{s}\right)\right) / C_{i}\right\}^{2}
$$

donde $\mathrm{C}_{\mathrm{i}}$ é a concentração da variável traçadora i na amostra de sedimento em suspensão; $\mathrm{P}_{\mathrm{s}}$ é a proporção de contribuição da fonte $\mathrm{s} ; \mathrm{C}_{\mathrm{si}}$ é o valor médio da variável traçadora i obtida na fonte s; e $Z_{s}$ é o fator de correção da granulometria para a fonte $\mathrm{s}$.

Segundo Walling \& Collins (2000) o modelo é adequado para a identificação das fontes se o erro relativo médio for menor que $15 \%$, em que resultados com erros relativos médios maiores que $15 \%$ devem ser excluídos. $\mathrm{O}$ erro relativo médio (ERM) é a média da soma dos quadrados dos resíduos de cada variável, fato importante visto que se pode avaliar individualmente a eficiência de cada variável dentro do modelo linear de otimização.

Os resultados estimados pelo modelo foram comparados 
com a variabilidade temporal e espacial das condições de uso do solo da bacia. Foram observadas na bacia, durante o período de monitoramento, as principais alterações, como os períodos de preparo do solo, plantio, colheita e pousio, as retificações de estradas e os aspectos hidrológicos; referidas informações possibilitam avaliar a sensibilidade e a eficácia do modelo multivariado na identificação de fontes, o tempo de residência e os efeitos do manejo do solo na emissão dos sedimentos erodidos na bacia hidrográfica. Por meio da compreensão da variabilidade espacial e temporal da emissão de sedimentos, os resultados obtidos subsidiarão a elaboração de medidas eficientes de controle da emissão de sedimentos para o manejo de bacias hidrográficas.

\section{RESULTADOS E DISCUSSÃO}

\section{Descrição do potencial discriminante das fontes}

Os resultados da análise discriminante apontam três diferentes fontes possíveis de serem discriminadas: lavoura (L), estrada (E) e pastagem (P). Considerando-se as outras fontes analisadas (pousio, lavouras recentes e calha fluvial) concluiu-se que não houve diferença significativa que as distinguisse das outras fontes. A fonte pousio não apresentou diferença com a fonte pastagem e as fontes lavouras recentes e calha fluvial não apresentaram diferença com a fonte lavoura. Na Tabela 3 se mostra o resultado do teste KruskalWallis considerando-se duas ou três fontes potenciais de sedimentos.

Tabela 3. Eficiência discriminante das variáveis químicas

\begin{tabular}{lccccc}
\hline \multirow{2}{*}{ Variável } & \multicolumn{2}{c}{$\begin{array}{c}\text { fontes } \\
\mathbf{L}+\mathbf{E}\end{array}$} & & \multicolumn{2}{c}{$\begin{array}{c}\text { 3 fontes } \\
\mathbf{L}+\mathbf{E}+\mathbf{P}\end{array}$} \\
\cline { 2 - 3 } \cline { 5 - 6 } C org & Valor de H & Valor de $\mathbf{p ~ ( \chi ^ { 2 } )}$ & & Valor de $\mathbf{H}$ & Valor de $\mathbf{p ~ ( \chi ^ { 2 } )}$ \\
$\mathrm{P}$ & 8,308 & $\underline{0,004}$ & & 10,19 & $\underline{0,006}$ \\
$\mathrm{~N}$ & 8,308 & $\underline{0,004}$ & & 14 & $\underline{0,001}$ \\
Mn tot & 7,964 & $\underline{0,005}$ & & 9,188 & $\underline{0,01}$ \\
Fe tot & 5,914 & $\underline{0,015}$ & & 8,378 & $\underline{0,015}$ \\
Mn dit & 5,769 & $\underline{0,016}$ & & 8,872 & $\underline{0,012}$ \\
Al oxal & 4,673 & $\underline{0,027}$ & & 6,771 & $\underline{0,034}$ \\
Na & 4,773 & $\underline{0,029}$ & & 8,329 & $\underline{0,016}$ \\
Al tot & 4,689 & $\underline{0,03}$ & & 6,7 & $\underline{0,035}$ \\
Fe dit & 3,692 & $\underline{0,055}$ & & 6,421 & $\underline{0,04}$ \\
Co & 3,692 & $\underline{0,055}$ & & 7,064 & $\underline{0,029}$ \\
Fe oxal & 3,169 & $\underline{0,075}$ & & 3,659 & 0,16 \\
Cr & 3,169 & $\underline{0,075}$ & & 12,31 & $\underline{0,002}$ \\
Al dit & 1,473 & 0,225 & & 2,057 & 0,358 \\
Zn & 1,095 & 0,295 & & 2,406 & 0,3 \\
Ca & 0,5211 & 0,47 & & 6,437 & 0,04 \\
\hline
\end{tabular}

(L) lavoura, (E) estrada e (P) pastagem; $\mathrm{H}$ crítico para 2 fontes $(95 \% ; 1 \mathrm{gl})=3,84 ; \mathrm{H}$ crítico para 3 fontes $(95 \% ; 2 g l)=5,99$

Através da determinação dos parâmetros Wilk's Lambda $\left(\Lambda^{*}\right)$, aproximação do teste $\mathrm{F}$ e distribuição Qui-quadrado $\left(\chi^{2}\right)$, os resultados da análise de variância multivariada determinaram o grupo de variáveis que otimiza a discriminação das fontes e diminui a dimensionalidade (Tabela 4). To- das as possibilidades de variáveis na análise de variância foram testadas e aquelas que apresentaram menor valor de $\Lambda^{*}$ e a significância para o teste $\mathrm{F}$ e $\chi^{2}$, foram escolhidas para serem utilizadas no modelo de classificação.

Tabela 4. Melhores composições das variáveis avaliadas

\begin{tabular}{|c|c|c|c|}
\hline Grupos & $\Lambda^{*}$ & Teste F $(0,05)$ & Qui-quadrado $\left(\chi^{2}\right)$ \\
\hline 3 fontes $(L+E+P)$ & & $F_{\text {crit } 12,20}=2,54$ & $\chi_{\text {crit }(95 \%, 12 \text { G.L.) }}^{2}=19,7$ \\
\hline $\mathrm{Fe}_{\text {tot' }^{\prime}} \mathrm{Fe}_{\text {oxal' }} \mathrm{Al}_{\text {oxal' }} \mathrm{Mn}_{\text {tot' }} \mathrm{Ca}, \mathrm{P}$ & 0,0057 & 20,34 & 64,51 \\
\hline 2 fontes $(L+E)$ & & $\mathrm{F}_{\text {crit } 6,5}=4,95$ & $\chi_{\text {crit (95\%, 6G.L.) }}^{2}=12,6$ \\
\hline $\mathrm{Fe}_{\text {tot' }} \mathrm{Fe}_{\text {oxal' }} \mathrm{Al}_{\text {oxal' }} \mathrm{Fe}_{\text {dit't }}, \mathrm{P}, \mathrm{Co}$ & 0,0563 & 13,97 & 20,14 \\
\hline $\mathrm{Fe}_{\text {tot' }^{\prime}} \mathrm{Fe}_{\text {oxal' }}, \mathrm{Al}_{\text {oxal }} \mathrm{Mn}_{\text {dit' }^{\prime}}, \mathrm{P}, \mathrm{Co}$ & 0,04281 & 18,63 & 22,06 \\
\hline
\end{tabular}

Além de se encontrar o conjunto que minimizasse o valor do parâmetro $\Lambda^{*}$ espera-se, ainda, que este conjunto represente diferentes processos associados à erosão hídrica e ao transporte dos sedimentos, em que as variáveis devem reproduzir mecanismos diferentes que ocorrem nas fontes, no que se refere, por exemplo, ao intemperismo, condições de redox e manejo do solo. Este objetivo foi alcançado, o conjunto proposto apresenta variáveis associadas ao manejo do solo $(\mathrm{P}$ e $\mathrm{Ca})$, processos de intemperismo e condições de redox (óxidos de $\mathrm{Fe}, \mathrm{Mn}$ e $\mathrm{Al}$ ) e à presença de metais $\left(\mathrm{Fe}_{\mathrm{tot}}\right.$, $\mathrm{Mn}_{\text {tot }}$ e Co).

As variáveis carbono orgânico (COT) e nitrogênio total (N) não foram utilizadas como variáveis traços, apesar de selecionadas na análise discriminante; a concentração desses elementos nos sedimentos em suspensão apresentou enriquecimento acima do qual os fatores de correção são capazes de corrigir; a hipótese provável é que o aporte de adubos solúveis e os dejetos animais lançados na rede de drenagem aumentam a adsorsão de $\mathrm{N}$ e COT nos sedimentos, além da variabilidade temporal de produção de biomassa.

\section{Classificação das amostras de sedimentos em suspensão}

Os resultados da classificação dos sedimentos em suspensão, considerando-se três fontes de sedimentos (lavoura, estrada e pastagem) foram insatisfatórios. Os erros relativos médios (ERM) foram maiores que 54\% para todos os eventos. As contribuições estimadas pelo modelo foram: $0,8 \%$ para as lavouras, $21,3 \%$ para as estradas e $77,9 \%$ para as pastagens. $\mathrm{O}$ alto valor do ERM demonstra que o programa Excel-Solver foi incapaz de encontrar uma solução correta considerando três fontes de sedimentos; além disso, a magnitude das contribuições de cada fonte é inconsistente com o processo erosivo observado na bacia.

Os resultados da classificação dos sedimentos em suspensão, considerando-se duas fontes de sedimentos (lavoura e estrada) foram satisfatórios. Com o modelo de otimização, encontrou-se uma solução viável para todos os eventos, sendo que os erros relativos médios foram $7 \%$ (média dos eventos). Os resultados das contribuições relativas de cada fonte são apresentados na Figura 2. A média da contribuição das lavouras foi de $64 \%$ e das estradas, $36 \%$; e resultado é coerente com a observação dos processos erosivos na bacia, em que as lavouras e as estradas são fontes preponderantes na produção de sedimentos. 


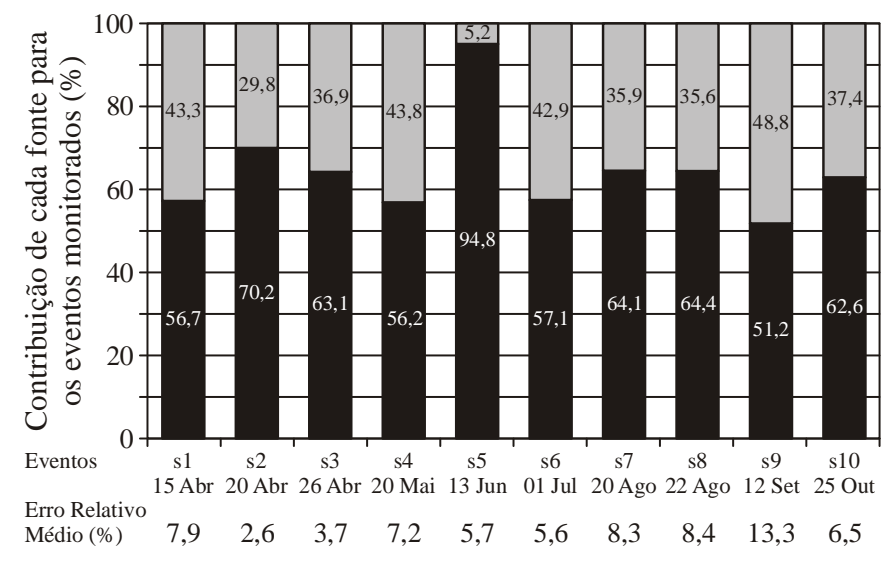

Figura 2. Resultados da contribuição relativa por evento das fontes lavoura (preto) e estrada (cinza), com os erros médios relativos

Observa-se grande variabilidade temporal na contribuição relativa de cada fonte. A média e o coeficiente de variação da fonte lavoura são, respectivamente, 64 e 19\%. Para as estradas, a média é $36 \%$ e o coeficiente de variação, $33 \%$; tal variabilidade confirma a hipótese de que a produção de sedimentos oscila conforme os fatores controladores de produção e a emissão de sedimentos durante o ano.

Observando-se a Figura 2 e se excluindo o evento s5 da análise, percebe-se uma clara oscilação na contribuição relativa de sedimentos pelas duas fontes. Após o mês de abril, a cobertura do solo aumenta, diminuindo a contribuição da fonte lavoura, eventos s2, s3 e s4; o evento s1 também seguiria esta tendência, porém se deu a retificação das estradas no início de abril, ocorrendo um aumento na contribuição das estradas e, consequientemente, a diminuição relativa das lavouras.

A partir do mês de agosto até setembro, as áreas de lavoura são lavradas e os resultados mostraram aumento gradativo na contribuição relativa das lavouras (s6, s7, s8); entretanto, no início de setembro ocorreu retificação das estradas e, novamente, um aumento na contribuição das estradas.

\section{CONCLUSÕES}

1. A utilização da técnica "fingerprinting" mostrou tratar-se de uma técnica adequada para a identificação das fontes de produção de sedimentos na bacia analisada.

2. O programa Excel-Solver foi capaz de encontrar uma solução viável quando duas fontes de sedimentos foram incorporadas na análise mas incapaz quando três fontes de sedimentos foram incorporadas à análise; verificou-se, então, a importância significativa das lavouras (64\%) e das estradas (36\%) na produção de sedimentos.

3. Notou-se, também, que a variação temporal da proporção com que cada fonte contribuiu na produção de sedimentos decorreu da alteração da cobertura vegetal da bacia e das obras de manutenção das estradas, durante o período de avaliação.

4. Entende-se, entretanto, que o aperfeiçoamento desse método deverá utilizar um programa matemático mais robusto para rodar o modelo de otimização e utilizar um número maior de elementos químicos de caráter conservativo e sensitivo às modificações causadas pelo manejo do solo.

\section{AGRADECIMENTOS}

Os autores agradecem ao hidrotécnico Adair Giongo, pelo valoroso apoio de campo; ao Programa RS-RURAL e aoCNPq (Conselho Nacional de Desenvolvimento Científico e Tecnológico).

\section{LITERATURA CITADA}

Carvalho, N. O. Hidrossedimentologia prática. Rio de Janeiro: CPRM. 1994. 372p.

Collins, A. L.; Walling, D. E. Selecting fingerprint properties for discriminating potential suspended sediment sources in river basins. Journal of Hydrology, v.261, n.1-4, p.218-244. 2002.

Collins, A. L.; Walling, D. E.; Leeks, G. J. L. Source type ascription for fluvial suspended sediment based on a quantitative composite fingerprinting technique. Catena, v.29, p.1-27. 1997.

FISP - Federal Interagency Sedimentation Project. The single-stage sampler for suspended sediment, Minneapolis, St. Anthony Falls Hydraulics Laboratory, 1961, p.01-105. Report 13

Horowitz, A. J. A primer on sediment trace element chemistry. 2th.ed. Chelsea: Lewis Publishers. 1991. 136p.

Inda Júnior, A. V. Caracterização da goethita e hematita em solos poligênicos. Porto Alegre: UFRGS, 2002. 126p. Tese Doutorado

Johnson, R. A.; Wichern, D. W. Applied multivariate statistical analysis. London: Prentice-Hall International. 1992. 642p.

Merten, G. H.; Minella, J. P. G. Projeto de monitoramento ambiental de microbacias hidrográficas - RS-Rural - Sub-Projeto 7 monitoramento hidrossedimentológico e da qualidade das águas. Instituto de Pesquisas Hidráulicas. Porto Alegre: UFRGS. 2003. 49p. Relatório Interno

Peart, M. R. Fingerprint sediment sources: An example from Hong Kong. In: Foster, I. D. L.; Gurnell, A. M.; Webb, B. W. (ed.) Sediment and water quality in river catchments. Chichester: John Wiley. 1995. p.179-186.

Symader, W.; Strunk, N. Determining the source of suspended particulate material. In: Walling, D. E.; Davies, T. R.; Hasholt, B. (ed.) Erosion, debris flow and environment in mountain regions. IAHS. Publ. v.209. p.177-185. 1992.

Tedesco, M. J.; Gianello, C.; Bissani, C. A.; Bohnen, H.; Volkweiss, S. J. Análises de solo, plantas e outros materiais. 2.ed. Porto Alegre: Departamento de Solos da UFRGS. 1995. 174p.

Walling, D. E.; Collins, A. L. Integrated assessment of catchment sediment budgets: A technical manual. Exeter: Department for International Development. 2000. 168p.

Walling, D. E.; Owens, P. N.; Leeks G. J. L. Fingerprint suspended sediment sources in the catchment of the River Ouse, Yorkshire, UK. Hydrological Processes, v.13, n.1, p.955-975. 1999.

Walling, D. E.; Woodward, J. C. Tracing sources of suspend sediment in river basins: A case study of the River Culm, Devon, UK. Marine Freshwater Resources, v.46, p.327-336. 1995.

Yu, L.; Oldfield, F. A multivariate mixing model for identifying sediment source from magnetic measurements. Quaternary Research, v.32, n.1, p.168-181. 1989. 